\title{
Capturing atomic-scale carrier dynamics with electrons
}

\author{
Peter Baum ${ }^{1,2}$ and Ferenc Krausz ${ }^{1,2}$ \\ ${ }^{1}$ Ludwig-Maximilians-Universität München, Am Coulombwall 1, 85748 Garching, Germany \\ ${ }^{2}$ Max-Planck-Institute of Quantum Optics, Hans-Kopfermann-Str. 1, 85748 Garching, Germany
}

Light-driven electronic motion unfolds on times as short as the cycle period of light and on length scales as small as the distance between two neighboring atoms in a molecule. Visualizing fundamental light-matter interactions therefore requires access to attosecond and picometer dimensions. Here we report on a potential unification of electron diffraction and microscopy with attosecond spectroscopy, which could provide a full space-time access to elementary electronic processes in matter and materials. We review recent progress in ultrafast diffraction and microscopy towards temporal resolutions approaching $10 \mathrm{fs}$ by use of state-of-the-art microwave technology and discuss our latest findings on all-optical compression approaches offering the potential for sub-femtosecond, sub-optical-cycle resolution. Four-dimensional electron diffraction with attosecond-picometer resolution will access all dynamics outside the atomic core, offering an all-embracing insight into fundamental electron-nuclear dynamics of complex materials. 


\section{$\underline{\text { Introduction }}$}

With Ahmed Zewail we lose an inspiring scientist and genuine pioneer of physics and chemistry at ultrashort time and length scales. He was among the first to unambiguously observe, together with others, the making and breaking of bonds during chemical reactions [1]. Early work concentrated on spectroscopic techniques, followed by a paradigm shift towards complete spacetime visualization via ultrafast electron diffraction and microscopy $[2,3]$. Both authors of this letter were highly inspired by Ahmed's positive and engulfing personality, by his optimism and strong aim for understanding complex materials. We are delighted that we have been given the opportunity to follow up his legacy in ultrafast electron diffraction and microscopy by advancing these techniques towards a novel level of application, namely from the domain of nuclear motion to that of carrier and electron dynamics. This letter shall review some recent contributions ${ }^{1}$ and also provide a forward-looking glimpse on the vivid perspectives we see lying ahead.

\section{$\underline{\text { Light-driven electronic motion }}$}

Almost any light-matter interaction starts with a periodic displacement of electron densities at lightwave frequencies and on dimensions defined by the atomic environment. Due to the threedimensional and anharmonic shape of inter-atomic potentials in most materials, this microscopic electronic motion is often complex and involves subtle spatio-temporal characteristics that lead to a rich range of macroscopic phenomena, for example the linear and nonlinear refractive index, light absorption and emission, frequency conversion, laser operation, photoelectric emission, lightenergy conversion or strong-field optical effects. Light-driven re-arrangement of the electronic subsystem is also the cause and driving force for the subsequent motion of the atoms, for example in photochemical reactions, ultrafast phase transformations, biomolecular energy transfers or medical diagnostics and therapy with light. A study of electronic motion in space and time with resolutions of attosecond and picometers is therefore appropriate to understand the very foundations of light-matter interaction and its consequences in the macroscopic domain.

Attosecond spectroscopy with laser-generated photon pulses $[4,5]$ has in the last several years opened up a direct view into light-driven electronic processes. The key technology is based on the controlled generation of single-light-cycle laser pulses with locked carrier-envelope phase or,

\footnotetext{
${ }^{1}$ References are intended as a starting point for the reader's own research. We apologize to all the pioneering scientists that could not be given proper credit for their groundbreaking contributions due to space limitations.
} 
more general, waveform-synthesized single-cycle or sub-cycle light fields. These electric fields allow inducing in a controlled way the electronic motion in atoms, gases or condensed matter, and at the same time provide via strong-field photoemission a source of attosecond extreme-UV pulses $[4,5]$ that are suitable for spectroscopic or streaking investigations of electron dynamics with suboptical-cycle time resolution.

In all these approaches, however, there remains a central restriction in spatial resolution. Attosecond XUV pulses have wavelengths not shorter than tens of nanometers, which is far away from atomic dimensions. An interesting possibility is to use the re-colliding electron wavefunction [6,7], but the offered femtosecond/Angstrom resolution combination comes at the cost of a strong entanglement between methodology and sample, restricting the applicability. The most ideal access to light-driven electron dynamics in complex materials and nanostructures would be direct and deliver attosecond and picometer resolution at the same time.

Electrons carry a rest mass and their de Broglie wavelength is therefore easily sub-atomic at energies of only tens of $\mathrm{keV}$. This fact is the basis of the atomic and sub-atomic resolution in electron microscopy and diffraction. Changes can in many cases even easier be recorded than static structures, because the basic diffraction pattern or image does not need an all-embracing interpretation. Early attempts to combine electron diffraction with picosecond and femtosecond temporal resolution have been held back by space charge effects and pump-probe synchronization issues. First pioneering studies by Mourou $[8,9]$ and Zewail $[10,11]$ provided picosecond resolution of structural dynamics in aluminum [9] and $\mathrm{CH}_{2} \mathrm{I}_{2}$ [11]. Ahmed Zewail and his team subsequently pushed this approach towards substantially higher temporal resolution and importantly, also towards much more complex materials [2, 3].

It was understood by Siwick and Miller [12] that space charge effects can be largely diminished by using very compact electron sources [12-14], where the propagation length between electron emission and probing is minimized to merely a few $\mathrm{mm}$. Alternatively it is possible to reduce the number of electrons per pulse to just hundreds [15], if reversible dynamics is investigated. These two approaches allowed entering the femtosecond domain of time resolution, first in melting experiments by Siwick and Miller [12] and later in femtosecond grazing-incidence diffraction from condensed-matter crystals and surfaces in the Zewail group [15, 16]. A central advantage of ultra-bright sources is the applicability to non-reversible processes in photochemistry 
[17], while the few-electron or ultimately single-electron [18, 19] approach (see below) has benefits for investigating electron dynamics $[20,21]$ or reversible condensed-matter physics $[15$, $16,22,23]$.

Siwick and Miller recognized that multi-electron pulses develop a surprisingly linear timeenergy correlation (chirp) under space-charge effects [24], which can in principle be reversed. The idea of using microwave compression of electron pulses for ultrafast diffraction was first mentioned by Fill et al. [25] and later demonstrated experientially by the Luiten group [26]. A key problem is laser-microwave synchronization [25], because the arrival time of the compressed electron bunch is following the phase of the microwave and not the timing of the pump laser. Various electronic lock schemes have therefore been developed; phase-locked loop electronics provides tens-of-femtoseconds jitter, time-stamping delivers about 30-fs (rms) resolution [27] and passive laser-repetition-rate enhancement [28] with time-of-flight-based drift control has recently provided a jitter of less than 5-fs (rms) from the Nyquist frequencies to tens of minutes [29].

Time resolution can alternatively be achieved with long electron pulses and post-selection, for example in the energy domain [30] or in spatially streaked diffraction patterns [31,32]. Electron pulses at $>\mathrm{MeV}$ central energy suffer less from space charge effects and are therefore useful for squeezing many electrons into extremely short pulses, although the de Broglie wavelength at $\mathrm{MeV}$ energies tends to become too short for practical atomic-scale diffraction [27].

There has also been important progress with electron pulse metrology. While first studies have inferred the time resolution from laser-triggered streak camera measurements $[11,18]$ there are now various higher-resolution techniques available, for example ponderomotive scattering [33], laser streaking [34] or terahertz deflection [35]. Among these techniques, laser streaking is unique in the sense that it works in the time-energy domain and hence provides not only the pulse duration but measures chirp, coherence and bandwidth as well [34]. In contrast, our recently developed terahertz deflection approach [35] does not require an energy analyzer and the method is therefore simpler in many experiments.

Ultrashort electron pulses can also be combined with electron microscopy in order to achieve real-space imaging of space-time dynamics $[18,36]$. Researchers either use single-short nanosecond imaging of irreversible processes [36] or pump-probe imaging with femtosecond resolution $[2,3]$. Ahmed Zewail and his team have pioneered the latter approach and studied in 
short time an impressive range of complex materials, for example DNA, nanostructures, correlated materials, liquid suspensions or fluids in carbon nanotubes. So far, ultrafast electron microscopy with femtosecond pulses has not reached genuine atomic resolution yet, mainly because the timeaveraged electron flux is still too low, but recent advances with needle sources or ultra-cold gas emitters in combination with aberration correctors seem to promise a realistic perspective [27].

\section{Single-electron pulses for attosecond imaging}

We considered in $2006[25,37]$ whether it would be possible to advance ultrafast electron diffraction and microscopy to the attosecond domain of time resolution. We thought that this endeavor would be rewarding, as it would provide a direct visualization of light-matter interaction unfolding on its fundamental space-time dimensions. It was clear that multi-electron pulses would suffer too much from space charge effects and uncorrelated phase-space heating to potentially deliver them onto a diffraction target at sub-femtosecond pulse duration. We therefore chose to work with single-electron pulses $[18,19]$ which are entirely immune to space charge effects and can therefore be manipulated into arbitrary space-time shape, as far as allowed by the uncertainty principle (and technical limitations) [27]. Our first proposals emerged, one based on microwave compression of single-electron wavepackets [25] and the other on the laser-based ponderomotive reshaping of a single-electron wavefunction into attosecond pulse trains [38]. It was immediately clear that experiments in the single-electron regime would require a substantially higher pumpprobe repetition rate than conventionally applied, but still low enough to allow the sample to relax between the excitations. Suitable lasers at high-kHz / low-MHz repetition rate were therefore developed, and a first experiment showed that pump-probe diffraction of reversible condensedmatter dynamics is indeed possible with single-electron pulses [23] if the excitation conditions are chosen carefully. We emphasize that our main aim with single-electron diffraction is to access light-driven motion of bound electrons in materials and/or collective electronic motion in nanophotonics, which are processes that are highly reversible even in the strong-field regime [39]. However, it is also possible to study atomic motions and irreversible processes such as chemical reactions, if the substance is replenished after each laser excitation. This task seems difficult but not impossible to solve, for example with substances in a gas jet or via laser desorption. In many cases at least a few (if not tens to hundreds) of electrons per pulse can still be tolerated in 
compressed pulses without substantially compromising the genuine single-electron physics in the beam [40].

\section{Phase-space control}

Even in the single-electron regime, where there are no space charge forces, a laser-emitted single-electron wave-function still lengthens in time [19]. This wavepacket spread originates from the dispersion of vacuum at non-relativistic energies in combination with the initial energy bandwidth of laser-emitted electron pulses. Photoemission at photon energies close to the work function [19] can alleviate this problem, but the initial energy bandwidth can never be reduced beyond the limit originating from the ultrafast emission duration due to the uncertainty principle [27]. Producing attosecond electron pulses at a target that is not extremely close to the emitter therefore requires wavepacket compression techniques even if no space charge is involved. We initially invoked a microwave compressor [25], similar to those systems usually applied for fighting against space charge [26], but optimized it for reshaping a single-electron wave-function in time [41]. We could reduce the timing jitter to a 5-fs level [29] and finally produce singleelectron pulses with a duration of 28 fs (full-width-at-half-maximum) or $12 \mathrm{fs}$ (rms) [40]. The spatial beam coherence at target was found sufficient to record atomic-resolution diffraction from complex organic molecular crystals or condensed-matter systems [40]. When proceeding towards even shorter pulses, microwave compression is better replaced or supplemented by an all-optical control approach [35]. Optically generated terahertz fields replace the microwaves and microstructured metal resonators or ultrathin foils substitute the pillbox cavity in providing a subwavelength-sized interaction region. Terahertz field cycles produced by optical rectification follow the timing of the generating laser pulses with a precision determined by the response time of the nonlinear electronic motion in the crystal. Consequently, there is a precise timing link between laser envelope and the terahertz field's zero crossing, exactly like required for controlling electron pulses in a jitter-free way. Without any feedback loop or beam stabilization system we demonstrated terahertz-compressed pulses with a timing drift of merely $3.7 \mathrm{fs}$ (rms) over several hours [35]. This encouraging result is a consequence of the all-optical control approach, where jitter and drifts only originate from mechanical instabilities of the optical arrangement. It is also possible to accelerate electron pulses to higher energy [42] or to drive photoemission at higher 
field strength [43]. There are also first results on optically controlling the electron beams in a transmission electron microscope [44], a development that might eventually allow pump-probe microscopy at ultimate temporal resolution and in real space.

\section{$\underline{\text { Towards attosecond electron pulses via direct or multi-stage compression }}$}

There are two key effects that limit the final pulse duration in an optically-driven compressor. First, the energy spread in the beam converts to timing spread on the path between compressor and target, lengthening the pulses. Second, nonlinearity of the optical field cycles on the time scale of the incoming pulse duration produces an imperfect temporal shape at target, because parts of the electron packet are compressed with non-identical strengths. Both effects scale favorably with a reduction of the compressor-target distance, but this in turn implies a higher temporal field gradient and higher peak intensity of the compression pulses. The longer the wavelength the more difficult is it to achieve high peak fields, because focus sizes scale with wavelength and nonlinear optical conversion efficiencies roughly follow the ratio of photon energies. Nevertheless, terahertz peak fields of $10^{10} \mathrm{~V} / \mathrm{m}$ have been demonstrated, which is more than $10^{3}$ times larger than applied in our initial work [35]. Substantially shorter distances of the temporal focus are therefore achievable, promising correspondingly shorter pulses.

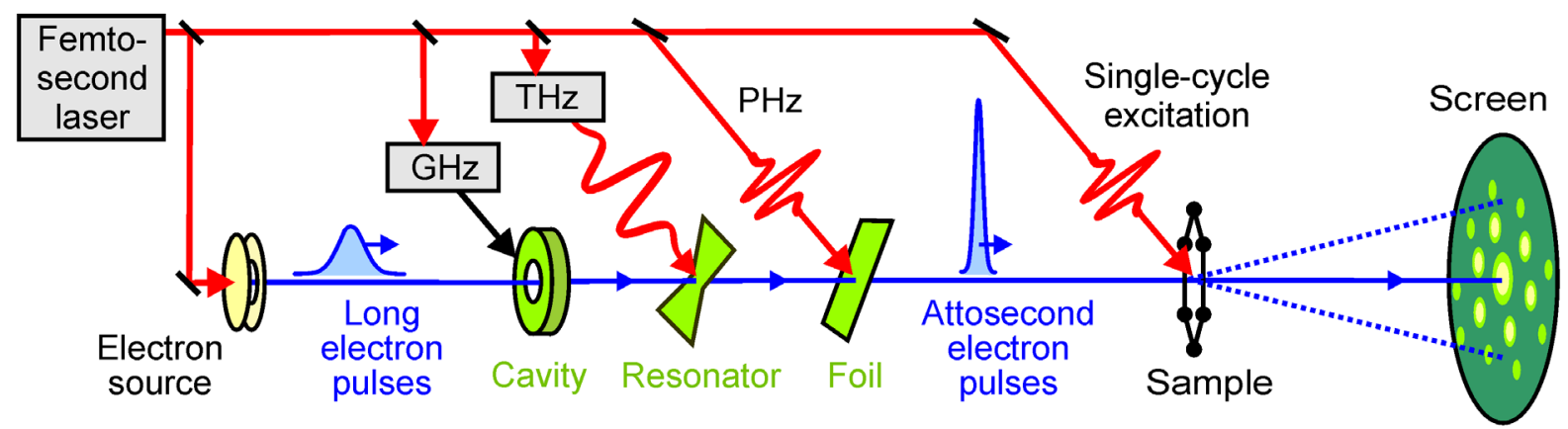

Fig.1. Space-time metrology with electrons. Shown is a concept for compressing electron pulses from nanosecond to attosecond duration over several compressor stages. Diffraction from a laser-excited sample can reveal any dynamics outside the atomic core with fundamental space-time resolution.

An alternative is the combination of several compression stages at increasing frequency [35]. Figure 1 shows a depiction of that concept at the example of three stages. A first stage is driven 
with microwaves, a second stage makes use of terahertz compression, and a final stage is operating with the optical cycles of the laser itself. In all stages, a structure of sub-wavelength size is required to break the symmetry of electron-field interaction for producing a net momentum exchange $[20,34]$. Omega-shaped cavities are ideal at microwave frequencies $(\mathrm{GHz})$, in the terahertz regime $(\mathrm{THz})$ it is possible to use micro-structured metal resonators, and at optical frequencies $(\mathrm{PHz})$ one can use an ultrathin metal membrane [34]. The membrane in the last stage is particularly appealing because it can be made essentially homogeneous over the electron beam profile and the interaction can be velocity-matched at suitable angle combinations [34]. If the incoming electron wavepacket is femtoseconds long, there will be a train of attosecond electron pulses [38], but if the incoming electron wavepacket is already pre-compressed to few-fs duration by the terahertz compressor, isolated attosecond electron pulses will occur. The final jitter level is determined solely by the timing of the last stage, where it is limited only by the ability to detect and correct optical interferences in the beam path.

\section{Towards attosecond electron microscopy and diffraction}

Given the noticeable progress of us and others towards few-femtosecond or attosecond electron pulses, it is appropriate to briefly discuss potential experiments of interest. Basically, almost any light-matter interaction unfolds at first as a light-driven motion of electron densities in the restoring potential provided by the atomic environment. This potential and therefore the electronic motions are typically complex and can involve multiple dimensions, nonlinearities and multi-electron effects. On slower time scales, complex atomic motion or molecular reactions can be caused by these electronic rearrangements via the changed inter-atomic forces they provide. With continuous electron beams, atomic-scale electron density distribution has been measured by diffraction [45], and femtosecond x-ray pulses could reveal cycle-averaged electronic displacements in $\mathrm{LiBH} 4$ and $\mathrm{KH}_{2} \mathrm{PO}_{4}$ when subjected to oscillating light cycles [46].

The feasibility of attosecond/Angstrom pump-probe diffraction with electron pulses was numerically investigated by teams lead by Manz [47], Starace [48], Baum [49] and others. It was found (within the applied approximations) that attosecond electron diffraction can indeed measure time-frozen charge densities and movies thereof. A problem is the strong static diffraction from nuclear cores and inner electrons because it is almost independent of the presence of laser cycles. 
Materials with rather low atomic weights are therefore easier to study [46-49]. Crystalline samples and every-electron detection are needed in the experiment, and the influence of shot noise and experimental imperfections to the overall integration time should be carefully estimated before conducting the investigation [50]. We note that single-cycle excitation pulses allow exposing a wide range of materials to peak field strength exceeding $1 \mathrm{~V} / \AA$, which is the typical field between atoms.

While the motion of bound electrons determines the essential physics in dielectrics, the function of semiconductors and metals is mainly defined by collective carrier dynamics. Elementary excitations, for example plasmons or polaritons, can have wavelengths in the fewnanometer regime. In order to see such fields in space and time one can invoke waveform electron microscopy [21] and record the time-dependent distortions of a sample's shadow pattern in order to reveal the local electromagnetic fields. Given attosecond electron pulses and a decent electron microscope with $1 \mathrm{~nm}$ resolution it will be possible to record any light-driven collective carrier motion on its fundamental length and time scales [21]. This may help clarifying the principles allowing or preventing nanophotonic information processing at lightwave frequencies. In principle, electron microscopy can have sub-atomic resolution, and real-space imaging of atomicscale electrodynamics might by possible, provided that aberration correctors can be found that deal with the few-eV bandwidth that attosecond electron pulses must have. In the mean time, diffraction still provides atomic resolution of crystalline materials.

\section{Conclusions}

It has taken many years to advance ultrafast electron diffraction and microscopy from first attempts to the huge range of applicability apparent today; this proliferation and development would not have been possible without the late Ahmed Zewail. Also, it has taken more than a decade to develop attosecond spectroscopy from the first discoveries to routine investigations of complex materials. We think that the unification of these two techniques, i.e. the development of few-femtosecond and maybe attosecond electron diffraction and microscopy, is a logical and valuable next step, in potentially delivering space-time access to any light-driven dynamics outside of the atomic core. Late Ahmed Zewail always pushed his co-workers into selecting the most complex available materials for their investigations. It will be interesting to see how broadly 
applicable the upcoming attosecond methodologies with electrons will become, but the huge versatility of modern electron microscopy leaves a lot of room for hope.

\section{Acknowledgements}

This work was supported by the ERC projects '4Dimaging' and 'DIVI', and by the DFG excellence cluster 'Munich Centre for Advanced Photonics'. We apologize for the incomplete nature of the reference list due to publisher limitations.

\section{$\underline{\text { References }}$}

[1] A. H. Zewail. Femtochemistry: Atomic-scale dynamics of the chemical bond using ultrafast lasers - (Nobel lecture). Angewandte Chemie-international Edition, 39(15):2587$2631,2000$.

[2] D. Shorokhov and A. H. Zewail. Perspective: 4D ultrafast electron microscopy-evolutions and revolutions. Journal of Chemical Physics, 144(8):080901, February 2016.

[3] B. Barwick and A. H. Zewail. Photonics and plasmonics in 4D ultrafast electron microscopy. ACS Photonics, 2(10):1391-1402, October 2015.

[4] P. M. Paul, E. S. Toma, P. Breger, G. Mullot, F. Auge, P. Balcou, H. G. Muller, and P. Agostini. Observation of a train of attosecond pulses from high harmonic generation. Science, 292(5522):1689-1692, June 2001.

[5] M. Hentschel, R. Kienberger, C. Spielmann, G. A. Reider, N. Milosevic, T. Brabec, P. Corkum, U. Heinzmann, M. Drescher, and F. Krausz. Attosecond metrology. Nature, 414(6863):509-513, November 2001.

[6] H. Niikura, F. Legare, R. Hasbani, A. D. Bandrauk, M. Y. Ivanov, D. M. Villeneuve, and P. B. Corkum. Sub-laser-cycle electron pulses for probing molecular dynamics. Nature, 417(6892):917-922, June 2002.

[7] C. I. Blaga, J. L. Xu, A. D. DiChiara, E. Sistrunk, K. K. Zhang, P. Agostini, T. A. Miller, L. F. DiMauro, and C. D. Lin. Imaging ultrafast molecular dynamics with laser-induced electron diffraction. Nature, 483(7388):194-197, March 2012.

[8] G. Mourou and S. Williamson. Picosecond electron-diffraction. Applied Physics Letters, 41(1):44-45, 1982. 
[9] S. Williamson, G. Mourou, and J. C. M. Li. Time-resolved laser-induced phasetransformation in aluminum. Physical Review Letters, 52(26):2364-2367, 1984.

[10] J. C. Williamson, M. Dantus, S. B. Kim, and A. H. Zewail. Ultrafast diffraction and molecular-structure. Chemical Physics Letters, 196(6):529-534, August 1992.

[11] J. C. Williamson, J. M. Cao, H. Ihee, H. Frey, and A. H. Zewail. Clocking transient chemical changes by ultrafast electron diffraction. Nature, 386(6621):159-162, March 1997.

[12] B. J. Siwick, J. R. Dwyer, R. E. Jordan, and R. J. D. Miller. An atomic-level view of melting using femtosecond electron diffraction. Science, 302(5649):1382-1385, November 2003.

[13] C. Gerbig, A. Senftleben, S. Morgenstern, C. Sarpe, and T. Baumert. Spatio-temporal resolution studies on a highly compact ultrafast electron diffractometer. New Journal of Physics, 17:043050, April 2015.

[14] L. Waldecker, R. Bertoni, and R. Ernstorfer. Compact femtosecond electron diffractometer with $100 \mathrm{kev}$ electron bunches approaching the single-electron pulse duration limit. Journal of Applied Physics, 117(4):UNSP 044903, January 2015.

[15] P. Baum, D. S. Yang, and A. H. Zewail. 4D visualization of transitional structures in phase transformations by electron diffraction. Science, 318(5851):788-792, November 2007.

[16] N. Gedik, D. S. Yang, G. Logvenov, I. Bozovic, and A. H. Zewail. Nonequilibrium phase transitions in cuprates observed by ultrafast electron crystallography. Science, 316(5823):425-429, April 2007.

[17] T. Ishikawa, S. A. Hayes, S. Keskin, G. Corthey, M. Hada, K. Pichugin, A. Marx, J. Hirscht, K. Shionuma, K. Onda, Y. Okimoto, S. Koshihara, T. Yamamoto, H. B. Cui, M. Nomura, Y. Oshima, M. Abdel-Jawad, R. Kato, and R. J. D. Miller. Direct observation of collective modes coupled to molecular orbital-driven charge transfer. Science, 350(6267):1501-1505, December 2015.

[18] V. A. Lobastov, R. Srinivasan, and A. H. Zewail. Four-dimensional ultrafast electron microscopy. Proceedings of the National Academy of Sciences of the United States of America, 102(20):7069-7073, May 2005.

[19] M. Aidelsburger, F. O. Kirchner, F. Krausz, and P. Baum. Single-electron pulses for ultrafast diffraction. Proceedings of the National Academy of Sciences of the United States of America, 107(46):19714-19719, November 2010. 
[20] B. Barwick, D. J. Flannigan, and A. H. Zewail. Photon-induced near-field electron microscopy. Nature, 462(7275):902-906, December 2009.

[21] A. Ryabov and P. Baum. Electron microscopy of electromagnetic waveforms. Science, 353(6297):374-377, July 2016.

[22] S. Wall, B. Krenzer, S. Wippermann, S. Sanna, F. Klasing, A. Hanisch-Blicharski, M. Kammler, W. G. Schmidt, and M. Horn-von Hoegen. Atomistic picture of charge density wave formation at surfaces. Physical Review Letters, 109(18):186101, November 2012.

[23] S. Lahme, C. Kealhofer, F. Krausz, and P. Baum. Femtosecond single-electron diffraction. Structural Dynamics, 1(3):034303, 2014.

[24] B. J. Siwick, J. R. Dwyer, R. E. Jordan, and R. J. D. Miller. Ultrafast electron optics: Propagation dynamics of femtosecond electron packets. Journal of Applied Physics, 92(3):1643-1648, August 2002.

[25] E. Fill, L. Veisz, A. Apolonski, and F. Krausz. Sub-fs electron pulses for ultrafast electron diffraction. New Journal of Physics, 8:272, November 2006.

[26] T. van Oudheusden, P. L. E. M. Pasmans, S. B. van der Geer, M. J. de Loos, M. J. van der Wiel, and O. J. Luiten. Compression of subrelativistic space-charge-dominated electron bunches for single-shot femtosecond electron diffraction. Physical Review Letters, 105(26):264801, December 2010.

[27] P. Baum. Towards ultimate temporal and spatial resolutions with ultrafast single-electron diffraction. Journal of Physics B-atomic Molecular and Optical Physics, 47(12):124005, June 2014.

[28] A. Gliserin, M. Walbran, and P. Baum. Passive optical enhancement of laser-microwave synchronization. Applied Physics Letters, 103(3):031113, July 2013.

[29] M. Walbran, A. Gliserin, K. Jung, J. Kim, and P. Baum. 5-femtosecond laser-electron synchronization for pump-probe crystallography and diffraction. Physical Review Applied, 4(4):044013, October 2015.

[30] P. Baum and A. Zewail. Femtosecond diffraction with chirped electron pulses. Chemical Physics Letters, 462(1-3):14-17, September 2008.

[31] M. Eichberger, N. Erasmus, K. Haupt, G. Kassier, A. von Flotow, J. Demsar, and H. Schwoerer. Femtosecond streaking of electron diffraction patterns to study structural dynamics in crystalline matter. Applied Physics Letters, 102(12):121106, March 2013. 
[32] C. M. Scoby, R. K. Li, E. Threlkeld, H. To, and P. Musumeci. Single-shot 35 fs temporal resolution electron shadowgraphy. Applied Physics Letters, 102(2):023506, January 2013.

[33] C. T. Hebeisen, G. Sciaini, M. Harb, R. Ernstorfer, T. Dartigalongue, S. G. Kruglik, and R. J. D. Miller. Grating enhanced ponderomotive scattering for visualization and full characterization of femtosecond electron pulses. Optics Express, 16(5):3334-3341, March 2008.

[34] F. O. Kirchner, A. Gliserin, F. Krausz, and P. Baum. Laser streaking of free electrons at 25 keV. Nature Photonics, 8(1):52-57, January 2014.

[35] C. Kealhofer, W. Schneider, D. Ehberger, A. Ryabov, F. Krausz, and P. Baum. All-optical control and metrology of electron pulses. Science, 352(6284):429-433, April 2016.

[36] T. LaGrange, M. R. Armstrong, K. Boyden, C. G. Brown, G. H. Campbell, J. D. Colvin, W. J. DeHope, A. M. Frank, D. J. Gibson, F. V. Hartemann, J. S. Kim, W. E. King, B. J. Pyke, B. W. Reed, M. D. Shirk, R. M. Shuttlesworth, B. C. Stuart, B. R. Torralva, and N. D. Browning. Single-shot dynamic transmission electron microscopy. Applied Physics Letters, 89(4):044105, July 2006.

[37] P. Baum and A. H. Zewail. Breaking resolution limits in ultrafast electron diffraction and microscopy. Proceedings of the National Academy of Sciences of the United States of America, 103(44):16105-16110, October 2006.

[38] P. Baum and A. H. Zewail. Attosecond electron pulses for 4D diffraction and microscopy. Proceedings of the National Academy of Sciences of the United States of America, 104(47):18409-18414, November 2007.

[39] M. Schultze, E. M. Bothschafter, A. Sommer, S. Holzner, W. Schweinberger, M. Fiess, M. Hofstetter, R. Kienberger, V. Apalkov, V. S. Yakovlev, M. I. Stockman, and F. Krausz. Controlling dielectrics with the electric field of light. Nature, 493(7430):75-78, January 2013.

[40] A. Gliserin, M. Walbran, F. Krausz, and P. Baum. Sub-phonon-period compression of electron pulses for atomic diffraction. Nature Communications, 6:8723, October 2015.

[41] A. Gliserin, A. Apolonski, F. Krausz, and P. Baum. Compression of single-electron pulses with a microwave cavity. New Journal of Physics, 14:073055, July 2012.

[42] E. A. Nanni, W. Q. R. Huang, K. H. Hong, K. Ravi, A. Fallahi, G. Moriena, R. J. D. Miller, and F. X. Kartner. Terahertz-driven linear electron acceleration. Nature Communications, 6:8486, October 2015. 
[43] W. Ronny Huang, Arya Fallahi, Xiaojun Wu, Huseyin Cankaya, Anne-Laure Calendron, Koustuban Ravi, Dongfang Zhang, Emilio A. Nanni, Kyung-Han Hong, and Franz X. Kärtner. Terahertz-driven, all-optical electron gun. Optica, 3(11):1209-1212, 2016.

[44] A. Feist, K. E. Echternkamp, J. Schauss, S. V. Yalunin, S. Schaefer, and C. Ropers. Quantum coherent optical phase modulation in an ultrafast transmission electron microscope. Nature, 521(7551):200, May 2015.

[45] J. M. Zuo, M. Kim, M. O'Keeffe, and J. C. H. Spence. Direct observation of d-orbital holes and $\mathrm{Cu}-\mathrm{Cu}$ bonding in Cu2O. Nature, 401(6748):49-52, September 1999.

[46] J. Stingl, F. Zamponi, B. Freyer, M. Woerner, T. Elsaesser, and A. Borgschulte. Electron transfer in a virtual quantum state of libh4 induced by strong optical fields and mapped by femtosecond x-ray diffraction. Physical Review Letters, 109(14):147402, October 2012.

[47] P. Baum, J. Manz, and A. Schild. Quantum model simulations of attosecond electron diffraction. Science China-physics Mechanics \& Astronomy, 53(6):987-1004, June 2010.

[48] H. C. Shao and A. F. Starace. Detecting electron motion in atoms and molecules. Physical Review Letters, 105(26):263201, December 2010.

[49] V. S. Yakovlev, M. I. Stockman, F. Krausz, and P. Baum. Atomic-scale diffractive imaging of sub-cycle electron dynamics in condensed matter. Scientific Reports, 5:14581, September 2015.

[50] C. Kealhofer, S. Lahme, T. Urban, and P. Baum. Signal-to-noise in femtosecond electron diffraction. Ultramicroscopy, 159:19-25, December 2015. 